\title{
SPECIAL $L$-VALUES AND PERIODS OF WEAKLY HOLOMORPHIC MODULAR FORMS
}

\author{
KATHRIN BRINGMANN, KARL-HEINZ FRICKE, AND ZACHARY A. KENT
}

(Communicated by Ken Ono)

\begin{abstract}
In this paper, we explore a method for associating $L$-series to weakly holomorphic modular forms and then proceed to study their $L$-values. As our main application, we prove a very curious limiting theorem which relates three "periods" of a mock modular form and its shadow to the ratio of their noncritical $L$-values. Critical $L$-values are then shown to fit nicely within the framework of period polynomials and an extended Eichler-Shimura theory recently studied by Guerzhoy, Ono, and the first and third authors.
\end{abstract}

\section{InTRODUCTION AND STATEMENT OF RESUlts}

Special values of $L$-series have been the subject of intense study in arithmetic algebraic geometry and analytic number theory. In particular, Kohnen and Zagier [13] and many others [6, 11] have pursued and made use of an underlying algebraic theory of special values of $L$-series associated to (holomorphic) modular forms. On the other hand, such values are central objects in the deep conjectures posed by Birch-Swinnerton-Dyer, Beilinson, and Bloch-Kato (see e.g. [1, 5, 7, 10, 14, 15. $19+21]$.

Here we consider special values of $L$-series associated to weakly holomorphic modular forms (those meromorphic modular forms whose only poles are supported at the cusps). The very existence of such values has proven elusive in the past due to the fact that the classical definitions in terms of infinite summation or integrals taken along the positive imaginary axis of the complex plane are divergent in this more general setting.

To precisely describe the problem we address in this paper, we briefly introduce the classical setting. Let $f(z)=\sum_{m=1}^{\infty} a_{f}(m) q^{m} \in S_{k}\left(q:=e^{2 \pi i z}\right.$ as usual $)$ be a holomorphic cusp form of weight $k \in 2 \mathbb{N}$ on $\mathrm{SL}_{2}(\mathbb{Z})$. Then for $\operatorname{Re}(s) \gg 0$, the $L$-series associated to $f$ is defined by

$$
L_{f}(s):=\sum_{m=1}^{\infty} \frac{a_{f}(m)}{m^{s}} .
$$

It is known that $L_{f}(s)$ satisfies a functional equation under $s \mapsto k-s$ and has an analytic continuation to the complex plane (which by abuse of notation we also denote by $\left.L_{f}(s)\right)$. The special values of $L_{f}(s)$ are those at integral arguments.

Received by the editors November 7, 2012.

2010 Mathematics Subject Classification. Primary 11F03, 11F11, 11F30, 11F37, 11F67.

The research of the first author was supported by the Alfried Krupp Prize for Young University Teachers of the Krupp Foundation. 
In particular, the critical values, those special values of $L_{f}(s)$ that lay within the critical strip $0<\operatorname{Re}(s)<k$, coincide with certain period integrals of $f$ as follows:

$$
L_{f}(n)=\frac{(2 \pi)^{n}}{(n-1) !} \int_{0}^{\infty} f(i y) y^{n-1} d y \quad \text { for } n=1,2, \ldots, k-1 .
$$

Moreover, when $f$ is a Hecke eigenform, Manin's Periods Theorem [16] states that the critical values are essentially algebraic integers. To be more precise, there exist two nonzero complex numbers $\omega_{f}^{ \pm}$, known as periods for $f$, such that the quotient $L_{f}^{*}(n) / \omega_{f}^{\operatorname{sgn}\left((-1)^{n+1}\right)}$ is an algebraic integer for $n=1,2, \ldots, k-1$. Now consider $f(z)=\sum_{m>m_{0}} a_{f}(m) q^{m} \in S_{k}^{!}$(with $m_{0}$ throughout the smallest integer s.t. $a_{f}\left(m_{0}\right) \neq 0$ ), where $S_{k}^{!}$denotes the space of weight $k$ weakly holomorphic cusp forms (i.e., those weakly holomorphic modular forms satisfying $a_{f}(0)=0$ ). In [2, Guerzhoy, Ono, and the first and third authors made many observations regarding critical values of holomorphic cusp forms by extending the classical theory of periods to the much larger space $S_{k}^{!}$. Clearly (1.1) and (1.2) are no longer welldefined for general forms in $S_{k}^{!}$due to their possible pole at $i \infty$ and the growth of their coefficients. To get around this issue, the authors of [2] use the formal Eichler integral given by

$$
\mathcal{E}_{f}(z):=\sum_{m \geq m_{0}} \frac{a_{f}(m)}{m^{k-1}} q^{m}
$$

rather than a period integral to develop their theory. In the case when $f \in S_{k}$, we have that

$$
\mathcal{E}_{f}(z)-z^{k-2} \mathcal{E}_{f}\left(\frac{-1}{z}\right)=\sum_{n=0}^{k-2} \frac{L_{f}(n+1)}{(k-2-n) !} \cdot(2 \pi i z)^{k-2-n} .
$$

In general, the left hand side of (1.4) is always defined for $f \in S_{k}^{!}$and always a polynomial in $z$. Therefore, as an interesting side effect, this suggests the existence of an $L$-series for weakly holomorphic cusp forms. Indeed, in $\S 2$, we introduce a way to regularize the period integrals in (1.2) so that they are well-defined for all forms in $S_{k}^{!}$. In doing so, we define $L$-series for $f \in S_{k}^{!}$and $t_{0}>0$ by

$$
\begin{aligned}
L_{f}(s) & :=\frac{(2 \pi)^{s}}{\Gamma(s)} L_{f}^{*}(s) \quad \text { with } \\
L_{f}^{*}(s) & :=\sum_{m \geq m_{0}} \frac{a_{f}(m) \Gamma\left(s, 2 \pi m t_{0}\right)}{(2 \pi m)^{s}}+i^{k} \sum_{m \geq m_{0}} \frac{a_{f}(m) \Gamma\left(k-s, \frac{2 \pi m}{t_{0}}\right)}{(2 \pi m)^{k-s}} .
\end{aligned}
$$

Here the incomplete gamma function, $\Gamma(s, z)$, is given by the analytic continuation (to an entire function with respect to $s$ and fixed $z \neq 0$ ) of $\int_{z}^{\infty} e^{-t} t^{s-1} d t$.

Remarks.

(1) For cusp forms the $L$-series $L_{f}^{*}(s)$ has been defined in the shape of (1.5) by Kohnen and Zagier [13] in order to carry out numerical calculations.

(2) Absolute convergence of $L_{f}^{*}(s)$ is guaranteed since $\Gamma(s, x) \sim x^{s-1} e^{-x}$ as $x \rightarrow \infty$, and the Circle Method implies that

$$
a_{f}(m) \ll m^{\frac{1}{2}\left(k-\frac{3}{2}\right)} e^{4 \pi \sqrt{m_{0} m}} .
$$


(3) Using the modularity of $f$, it is not hard to see that (1.5) is independent of $t_{0}$. Moreover, we find in Theorem 2.2 that $L_{f}^{*}(s)$ satisfies a functional equation under $s \mapsto k-s$ and has an integral representation.

Armed now with the $L$-series for $f \in S_{k}^{!}$, we are in a position to study their special values and their associated periods. In $\S 2$, we give a detailed account of regularized integration and how it relates to $L_{f}(s)$ and the formal Eichler integrals (1.3) of [2].

To state our first main result, we require harmonic weak Maass forms. The theory of harmonic weak Maass forms [3], for example, explains Ramanujan's mock theta functions 22 24]. Every harmonic weak Maass form $\mathcal{F}(z)$ has a unique natural decomposition

$$
\mathcal{F}(z)=\mathcal{F}^{-}(z)+\mathcal{F}^{+}(z),
$$

where $\mathcal{F}^{-}$(resp. $\mathcal{F}^{+}$) is nonholomorphic (resp. holomorphic) on the complex upper half-plane. The holomorphic part $\mathcal{F}^{+}$has a Fourier expansion

$$
\mathcal{F}^{+}(z)=\sum_{m \geq m_{0}} c_{\mathcal{F}}^{+}(m) q^{m}
$$

which, following Zagier, we call a mock modular form. The nonholomorphic part has an expansion in incomplete gamma functions. The differential operators $D^{k-1}$ with $D:=\frac{1}{2 \pi i} \cdot \frac{\partial}{\partial z}$, and $\xi_{2-k}:=2 i y^{2-k} \cdot \frac{\bar{\partial}}{\partial \bar{z}}$, which play a central role in the theory, only see the holomorphic and nonholomorphic parts, respectively, of such forms. Furthermore, in 3,4] it is shown that they define the surjective maps

$$
\xi_{2-k}:\left\{\begin{array}{l}
H_{2-k} \rightarrow M_{k}^{!}, \\
H_{2-k}^{*} \rightarrow S_{k}
\end{array} \quad \text { and } \quad D^{k-1}:\left\{\begin{array}{l}
H_{2-k} \longrightarrow M_{k}^{!}, \\
H_{2-k}^{*} \longrightarrow S_{k}^{!},
\end{array}\right.\right.
$$

where $M_{k}^{!}$denotes the space of weight $k$ weakly holomorphic modular forms, $H_{2-k}$ is the space of harmonic weak Maass forms of weight $2-k$, and $H_{2-k}^{*} \subseteq H_{2-k}$ is the subspace of harmonic weak Maass forms of weight $2-k$ whose image under $\xi_{2-k}$ maps onto $S_{k}$.

Following Proposition 5.1 of 4, we are always able to find a harmonic weak Maass form $\mathcal{F} \in H_{2-k}^{*}$ which is good for a normalized Hecke eigenform $g \in S_{k}$ (see $\S 3$ for the definition). In general, the coefficients of the corresponding $\mathcal{F}^{+}$appear to be transcendental. However, in [9], Guerzhoy, Ono, and the third author proved that the coefficients of the corrected form, $D^{k-1}\left(\mathcal{F}^{+}\right)-c_{\mathcal{F}}^{+}(1) g$, are in $K_{g}$ (where $\left.K_{g}=\mathbb{Q}\left(a_{g}(1), a_{g}(2), \ldots\right)\right)$. This coefficient, $c_{\mathcal{F}}^{+}(1)$ of $\mathcal{F}^{+}$, is considered to be a third period associated to the eigenform $g \in S_{k}$. It turns out (see Corollary 4.4) that there is a nice relation between the $L$-series from $D^{k-1}(\mathcal{F})$ and $\xi_{2-k}(\mathcal{F})$ inside the critical strip. Numerical computations show that such a result is not true at integers outside the critical strip. However, surprisingly, the quotient of the two $L$-values has some interesting limiting behavior as $n \rightarrow \infty$, which relates the three periods of an eigenform $g \in S_{k}$.

Theorem 1.1. Let $g \in S_{k}$ be a normalized Hecke eigenform with periods $\omega_{g}^{ \pm}$chosen such that $\langle g, g\rangle=\omega_{g}^{+} \omega_{g}^{-}$, where $\langle g, g\rangle$ is the usual Peterson inner product. Let $\mathcal{F} \in H_{2-k}^{*}$ be good for $g$. Then for $\delta=0$ or 1 we have that

$$
\lim _{\substack{n \rightarrow \infty \\ n \rightarrow \bmod 2)}} \frac{L_{D^{k-1}(\mathcal{F})}(n)}{L_{\xi_{2-k}(\mathcal{F})}(n)}=\left(c_{\mathcal{F}}^{+}(1)+(-1)^{\delta} c_{\mathcal{F}}^{+}(-1)\right) \omega_{g}^{+} \omega_{g}^{-} .
$$


Remarks.

(1) By a theorem of Kohnen and Zagier (p. 202 of [12]), we may always choose $\omega_{g}^{ \pm}$such that $\langle g, g\rangle=\omega_{g}^{+} \omega_{g}^{-}$for a normalized Hecke eigenform $g \in S_{k}$.

(2) One can prove an analogous result for twisted $L$-series as defined in (1.7).

As our other application, we extend a result of 2 which shows that forms $\mathcal{F} \in H_{2-k}^{*}$ encode the critical values of $L$-functions arising from $S_{k}$ to the weakly holomorphic setting.

We also define twisted $L$-series associated to forms in $S_{k}^{!}$. First recall the situation for cusp forms $f \in S_{k}$. For a Dirichlet character $\chi$ with conductor $c$, the $L$-series of $f$ twisted by $\chi$ is

$$
L_{f}(\chi, s):=\sum_{m=1}^{\infty} \frac{\chi(m) a_{f}(m)}{m^{s}} .
$$

We define the associated twisted $L$-series, for $t_{0}>0$, by

$$
L_{f}(\chi, s):=\frac{(2 \pi)^{s}}{\Gamma(s)} L_{f}^{*}(\chi, s)
$$

with

$L_{f}^{*}(\chi, s):=\sum_{m \geq m_{0}} \frac{\chi(m) a_{f}(m) \Gamma\left(s, 2 \pi m t_{0}\right)}{(2 \pi m)^{s}}+i^{k} c^{k-2 s} \sum_{m \geq m_{0}} \frac{\chi(-m) a_{f}(m) \Gamma\left(k-s, \frac{2 \pi m}{c^{2} t_{0}}\right)}{(2 \pi m)^{k-s}}$.

Moreover, for $\gamma=\left(\begin{array}{ll}a & b \\ c & d\end{array}\right) \in \mathrm{SL}_{2}(\mathbb{Z})$, define the $\gamma$-mock modular period function for $\mathcal{F}^{+}$by

$$
\mathbb{P}\left(\mathcal{F}^{+}, \gamma ; z\right):=\frac{(4 \pi)^{k-1}}{(k-2) !}\left(\mathcal{F}^{+}-\left.\mathcal{F}^{+}\right|_{2-k} \gamma\right)(z),
$$

where for any function $g: \mathbb{H} \rightarrow \mathbb{C}$, we let $\left.g\right|_{\ell} \gamma(z):=(c z+d)^{-\ell} g\left(\frac{a z+b}{c z+d}\right)$. For a positive integer $c$, let $\zeta_{c}:=e^{2 \pi i / c}$, and for $0 \leq d<c$, let $\gamma_{c, d} \in \mathrm{SL}_{2}(\mathbb{Z})$ be any matrix satisfying $\gamma_{c, d}:=\left(\begin{array}{cc}* & * \\ c^{\prime} & d^{\prime}\end{array}\right)$. Here the integers $0 \leq d^{\prime}<c^{\prime}$ are chosen so that $\frac{d}{c}=\frac{d^{\prime}}{c^{\prime}}$ in lowest terms. We then obtain

Theorem 1.2. Let $\mathcal{F} \in H_{2-k}$ such that $f:=\xi_{2-k}(\mathcal{F}) \in S_{k}^{!}$. Then we have that

$$
\overline{\mathbb{P}\left(\mathcal{F}^{+}, \gamma_{1,0} ; \bar{z}\right)}=\sum_{n=0}^{k-2} \frac{L_{f}(n+1)}{(k-2-n) !}(2 \pi i z)^{k-2-n} .
$$

Moreover, if $\chi$ is a Dirichlet character with conductor $c$, then

$$
\frac{1}{c} \sum_{\ell(\bmod c)^{*}} \chi(\ell) \sum_{d=0}^{c-1} \zeta_{c}^{\ell d} \cdot \overline{\mathbb{P}\left(\mathcal{F}^{+}, \gamma_{c, d} ; \bar{z}-\frac{d}{c}\right)}=\sum_{n=0}^{k-2} \frac{L_{f}(\chi, n+1)}{(k-2-n) !}(2 \pi i z)^{k-2-n},
$$

where the sum on $\ell$ runs over those integers coprime to $c$.

Remark. Theorem 1.2 was proven in [2] in the special case when $\xi_{2-k}(\mathcal{F}) \in S_{k}$.

In $\S 2$, we define regularized $L$-series and show that they have an integral representation. We also show (see Theorem 2.4) that the error of modularity of an Eichler integral may be viewed as the generating function of critical $L$-values. We also prove certain vanishing results for a subclass of weakly holomorphic forms. 
In $\S 3$, we recall basic facts regarding the theory of harmonic weak Maass forms and the extended Eichler-Shimura theory of [2]. In $\S 4$, we discuss various ways to twist the $L$-series and derive related results including Theorem 1.2 . In $\S 5$, we prove Theorem 1.1.

\section{Regularized $L$-SERIES FOR $S_{k}^{!}$}

In this section we describe how to associate $L$-series to weakly holomorphic cusp forms. Let us start by recalling what is known for classical cusp forms.

Theorem 2.1. If $f \in S_{k}$, we have for $s \gg 0$ the integral representation

$$
L_{f}^{*}(s)=\int_{0}^{\infty} f(i y) y^{s-1} d y .
$$

Furthermore, $L_{f}^{*}$ has a holomorphic continuation to $\mathbb{C}$ and we have the functional equation

$$
L_{f}^{*}(k-s)=i^{k} L_{f}^{*}(s) .
$$

To generalize these results to the setting of weakly holomorphic forms, we require certain regularized integrals. For this, consider a continuous function $f: \mathbb{H} \rightarrow \mathbb{C}$. Assume that there is a $c \in \mathbb{R}^{+}$such that

$$
f(z)=O\left(e^{c \operatorname{Im}(z)}\right)
$$

uniformly in $\operatorname{Re}(z)$ as $\operatorname{Im}(z) \rightarrow \infty$. Then, for each $z_{0} \in \mathbb{H}$, the integral

$$
\int_{z_{0}}^{i \infty} e^{u i w} f(w) d w
$$

(where the path of integration lies within a vertical strip) is convergent for $u \in \mathbb{C}$ with $\operatorname{Re}(u) \gg 0$. If this integral has an analytic continuation to $u=0$, we define the regularized integral by

$$
R . \int_{z_{0}}^{i \infty} f(w) d w:=\left[\int_{z_{0}}^{i \infty} e^{u i w} f(w) d w\right]_{u=0},
$$

where the right hand side means that we take the value at $u=0$ of the analytic continuation of the integral. Similarly, we define integrals at other cusps $\mathfrak{a}$. Specifically, suppose that $\mathfrak{a}=\sigma_{\mathfrak{a}}(i \infty)$ for a scaling matrix $\sigma_{\mathfrak{a}} \in \mathrm{SL}_{2}(\mathbb{Z})$. If $f\left(\sigma_{\mathfrak{a}} z\right)$ satisfies (2.1), then we define

$$
R \cdot \int_{z_{0}}^{\mathfrak{a}} f(w) d w:=\left.R \cdot \int_{\sigma_{\mathfrak{a}}^{-1} z_{0}}^{i \infty} f\right|_{2} \sigma_{a}(w) d w .
$$

For cusps $\mathfrak{a}, \mathfrak{b}$, we set

$$
R \cdot \int_{\mathfrak{a}}^{\mathfrak{b}} f(w) d w:=R \cdot \int_{z_{0}}^{\mathfrak{b}} f(w) d w+R \cdot \int_{\mathfrak{a}}^{z_{0}} f(w) d w
$$

for any $z_{0} \in \mathbb{H}$. It is not hard to see that this integral is independent of $z_{0} \in \mathbb{H}$. We now prove an analogue to Theorem 2.1 which will also appear in the second author's thesis.

Theorem 2.2. Assume that $f \in S_{k}^{!}$. We have the identity

$$
L_{f}^{*}(s)=R \cdot \int_{0}^{\infty} f(i y) y^{s-1} d y .
$$


Furthermore, $L_{f}^{*}(s)$ satisfies the functional equation

$$
L_{f}^{*}(k-s)=i^{k} L_{f}^{*}(s) .
$$

Proof. To prove (2.3), we split the integral into two ranges. Inserting the Fourier expansion of $f(z)=\sum_{m \geq m_{0}} a_{f}(m) q^{m}$ yields that, for arbitrary $t_{0}>0$, we have

$$
\begin{aligned}
R . \int_{t_{0}}^{\infty} f(i y) y^{s-1} d y & =\sum_{m \geq m_{0}} a_{f}(m)\left[\int_{t_{0}}^{\infty} e^{-2 \pi m y-t y} y^{s-1} d y\right]_{t=0} \\
& =\left[\sum_{m \geq m_{0}} \frac{a_{f}(m)}{(t+2 \pi m)^{s}} \int_{2 \pi m t_{0}+t t_{0}}^{\infty} e^{-y} y^{s-1} d y\right]_{t=0} \\
& =\sum_{m \geq m_{0}} \frac{a_{f}(m)}{(2 \pi m)^{s}} \Gamma\left(s, 2 \pi m t_{0}\right) .
\end{aligned}
$$

Similarly we compute that

$$
R . \int_{0}^{t_{0}} f(i y) y^{s-1} d y=i^{-k} \sum_{m \geq m_{0}} \frac{a_{f}(m) \Gamma\left(k-s, \frac{2 \pi m}{t_{0}}\right)}{(2 \pi m)^{k-s}} .
$$

This directly yields (2.3). Moreover, the functional equation follows directly from the independence of $t_{0}$.

Recalling the Eichler integral (1.3), we find that if $f \in S_{k}$, then it is not hard to see that

$$
\mathcal{E}_{f}(z)=c_{k}^{-1} \int_{z}^{i \infty} f(\tau)(z-\tau)^{k-2} d \tau
$$

where $c_{k}:=-\frac{(k-2) !}{(2 \pi i)^{k-1}}$. We show that a similar result holds when $f \in S_{k}^{!}$.

Lemma 2.3. For $f \in S_{k}^{!}$, we have

$$
\mathcal{E}_{f}(z)=c_{k}^{-1} R \cdot \int_{z}^{i \infty} f(\tau)(\tau-z)^{k-2} d \tau .
$$

Proof. In the range of integration, the integrand has only a possible pole when $\tau \rightarrow i \infty$. Thus we compute, writing $f(z)=\sum_{m \geq m_{0}} a_{f}(m) q^{m}$,

$$
\begin{aligned}
R . \int_{z}^{i \infty} f(\tau)(\tau-z)^{k-2} d \tau & =\left[\int_{z}^{i \infty} e^{i u \tau} f(\tau)(\tau-z)^{k-2} d \tau\right]_{u=0} \\
& =\sum_{m \geq m_{0}} a_{f}(m)\left[\int_{z}^{i \infty} e^{i u \tau+2 \pi i m \tau}(\tau-z)^{k-2} d \tau\right]_{u=0} \\
& =\sum_{m \geq m_{0}} a_{f}(m) q^{m}\left[\int_{0}^{i \infty} e^{i \tau(u+2 \pi m)} \tau^{k-2} d \tau\right]_{u=0} .
\end{aligned}
$$

The integral now converges at $u=0$, and inserting the integral representation of the gamma function yields the claim.

We next consider the "error of modularity" of Eichler integrals. To be more precise, let

$$
r(f ; z):=c_{k}\left(\mathcal{E}_{f}(z)-\left.\mathcal{E}_{f}(z)\right|_{2-k} S\right)
$$


with $S:=\left(\begin{array}{cc}0 & -1 \\ 1 & 0\end{array}\right)$. It is known [2,12] that $r(f ; z)$ satisfies period relations, i.e.,

$$
r(f ; z) \in W:=\left\{P \in V_{k-2}: P+\left.P\right|_{2-k} S=P+\left.P\right|_{2-k} U+\left.P\right|_{2-k} U^{2}=0\right\},
$$

where $U:=\left(\begin{array}{cc}1 & -1 \\ 1 & 0\end{array}\right)$ and $V_{k-2}$ denotes the space of polynomials of degree at most $k-2$. We next prove that the period polynomial $r(f ; z)$ represents the generating functions for $L$-values inside the critical strip (see [2] for the case of classical cusp forms).

Theorem 2.4. We have that for $f \in S_{k}^{!}$,

$$
r(f ; z)=\sum_{n=0}^{k-2} i^{1-n}\left(\begin{array}{c}
k-2 \\
n
\end{array}\right) L_{f}^{*}(n+1) z^{k-2-n} .
$$

Proof. Making the change of variables $\tau \rightarrow-\frac{1}{\tau}$ in the second summand defining $r(f ; z)$ and applying Lemma 2.3 easily gives that

$$
r(f ; z)=R . \int_{0}^{i \infty} f(\tau)(\tau-z)^{k-2} d \tau .
$$

Now the claim follows by using the binomial expansion, (1.5) and (2.3).

As an application, we next turn to some simple vanishing results of regularized $L$-values associated to forms in $D^{k-1}\left(M_{2-k}^{!}\right)$which play an important role in the Hecke theory for weakly holomorphic forms [19].

Theorem 2.5. If $f \in D^{k-1}\left(M_{2-k}^{!}\right)$, then we have for $2 \leq n \leq k-2$ that

$$
L_{f}(n)=0 \text {. }
$$

Moreover, $L_{f}(1)=L_{f}(k-1)=0$ if and only if $f \in D^{k-1}\left(S_{2-k}^{!}\right)$.

Proof. Assume $f=D^{k-1}(F)$ with $F \in M_{2-k}^{!}$. We have that

$$
\mathcal{E}_{f}=F+c,
$$

where $c$ is the negative of the constant term of $F$. Using the fact that $F \in M_{2-k}^{!}$ gives that

$$
\mathcal{E}_{f}(z)-z^{k-2} \mathcal{E}_{f}\left(-\frac{1}{z}\right)=c\left(1-z^{k-2}\right) .
$$

From this we obtain the claim by Theorem 2.4 .

\section{HARMONIC WEAK MAASS FORMS}

We let $z=x+i y \in \mathbb{H}$, with $x, y \in \mathbb{R}$, and suppose throughout that $k \geq 4$ is even. The weight $2-k$ hyperbolic Laplacian is defined by

$$
\Delta_{2-k}:=-y^{2}\left(\frac{\partial^{2}}{\partial x^{2}}+\frac{\partial^{2}}{\partial y^{2}}\right)+i(2-k) y\left(\frac{\partial}{\partial x}+i \frac{\partial}{\partial y}\right) .
$$

A harmonic (weak) Maass form of weight $2-k$ is any smooth function $\mathcal{F}: \mathbb{H} \rightarrow \mathbb{C}$ satisfying:

(i) $\mathcal{F}(z)=\left.F\right|_{2-k} \gamma(z)$ for all $\gamma=\left(\begin{array}{ll}a & b \\ c & d\end{array}\right) \in S L_{2}(\mathbb{Z})$.

(ii) $\Delta_{2-k}(\mathcal{F})=0$.

(iii) The function $\mathcal{F}$ has at most linear exponential growth at infinity. 
We denote the space of such forms by $H_{2-k}$. Moreover, we let $H_{2-k}^{*} \subset H_{2-k}$ be the subspace of forms that are mapped to $S_{k}$ under $\xi_{2-k}$.

Every harmonic Maass form $\mathcal{F}$ has two natural associated period polynomials, one coming from $\xi_{2-k}(\mathcal{F})$ and one from $D^{k-1}(\mathcal{F})$. The next theorem from [2] shows that these are related.

Theorem 3.1. If $\mathcal{F} \in H_{2-k}$, then we have that

$$
r\left(\xi_{2-k}(\mathcal{F}) ; z\right) \equiv-\frac{(4 \pi)^{k-1}}{(k-2) !} \cdot \overline{r\left(D^{k-1}(\mathcal{F}) ; \bar{z}\right)} \quad\left(\bmod z^{k-2}-1\right),
$$

where equivalence modulo $z^{k-2}-1$ means that the difference of the two functions is a constant multiple of $z^{k-2}-1$. Moreover, there is a function $\widehat{\mathcal{F}} \in H_{2-k}$ for which $\xi_{2-k}(\widehat{\mathcal{F}})=\xi_{2-k}(\mathcal{F})$ and

$$
r\left(\xi_{2-k}(\mathcal{F}) ; z\right)=-\frac{(4 \pi)^{k-1}}{(k-2) !} \cdot \overline{r\left(D^{k-1}(\widehat{\mathcal{F}}) ; \bar{z}\right)} .
$$

This directly relates the $L$-values associated to the two pieces of a harmonic Maass form.

Corollary 3.2. If $\mathcal{F} \in H_{2-k}$, then for integers $0<n<k-2$, we have that

$$
L_{\xi_{2-k}(\mathcal{F})}(n+1)=(-1)^{n} \frac{(4 \pi)^{k-1}}{(k-2) !} \overline{L_{D^{k-1}(\mathcal{F})}(n+1)} .
$$

Finally we need the notation of good. We say that a harmonic Maass form $\mathcal{F} \in H_{2-k}$ is good for the normalized Hecke eigenform $g(z):=\sum_{n=1}^{\infty} a_{g}(n) q^{n} \in S_{k}$ if it satisfies the following:

(1) The principal part of $\mathcal{F}$ at the cusp $i \infty$ belongs to $K_{g}\left[q^{-1}\right]$, where $K_{g}:=$ $\mathbb{Q}\left(a_{g}(1), a_{g}(2), \ldots\right)$.

(2) We have that $\xi_{2-k}(\mathcal{F})=\frac{g}{\langle g, g\rangle}$.

\section{TWISTED $L$-SERIES}

Throughout this section, we let $\chi$ be a Dirichlet character with conductor $c$. We require, as an auxiliary function, a regularized $L$-series asscociated to $f(z)=$ $\sum_{m \geq m_{0}} a_{f}(m) q^{m} \in S_{k}^{!}$twisted by roots of unity. To be more precise, for $(d, c)=1$, we define, for $t>0$

$$
\begin{aligned}
L_{f}\left(\zeta_{c}^{d}, s\right):= & \frac{(2 \pi)^{s}}{\Gamma(s)} L_{f}^{*}\left(\zeta_{c}^{d}, s\right) \quad \text { with } \\
L_{f}^{*}\left(\zeta_{c}^{d}, s\right):= & \sum_{m \geq m_{0}} \frac{a_{f}(m) \zeta_{c}^{d m} \Gamma\left(s, 2 \pi m t_{0}\right)}{(2 \pi m)^{s}} \\
& +i^{k} c^{k-2 s} \sum_{m \geq m_{0}} \frac{a_{f}(m) \zeta_{c}^{-a m} \Gamma\left(k-s, \frac{2 \pi m}{c^{2} t_{0}}\right)}{(2 \pi m)^{k-s}},
\end{aligned}
$$

where $a$ is defined by $a d \equiv 1(\bmod c)$. As before we can see that this definition is independent of $t_{0}$. A straightforward calculation allows us to write the $L$-series twisted by $\chi$ in terms of the above defined $L$-series. 
Lemma 4.1. We have that

$$
L_{f}^{*}(\chi, s)=\frac{1}{c} \sum_{\ell} \chi(\ell) \sum_{d=0}^{c-1} \zeta_{c}^{-\ell d} L_{f}^{*}\left(\zeta_{c}^{d}, s\right) .
$$

A calculation completely analogous to that of Theorem 2.2 yields the following:

Theorem 4.2. Assume that $f \in S_{k}^{!}$. We have the integral representation

$$
L_{f}^{*}\left(\zeta_{c}^{d}, s\right)=i^{-s} R . \int_{\frac{d}{c}}^{i \infty} f(w)\left(w-\frac{d}{c}\right)^{s-1} d w .
$$

Moreover, it satisfies the functional equation

$$
L_{f}^{*}\left(\zeta_{c}^{d}, k-s\right)=i^{k} c^{2 s-k} L_{f}^{*}\left(\zeta_{c}^{-a}, s\right) .
$$

Clearly, by Lemma 4.1 we also obtain an integral representation and functional equation for the twisted $L$-series. Our next result concerns a twisted analogue of Corollary [3.2, relating the two $L$-functions naturally associated to a harmonic Maass form.

Theorem 4.3. Let $\mathcal{F} \in H_{2-k}$ with $\xi_{2-k}(\mathcal{F}), D^{k-1}(\mathcal{F}) \in S_{k}^{!}$. Then we have for $0<n<k-2$

$$
L_{\xi_{2-k}(\mathcal{F})}\left(\zeta_{c}^{d}, n+1\right)=(-1)^{n} \frac{(4 \pi)^{k-1}}{(k-2) !} \overline{L_{D^{k-1}(\mathcal{F})}\left(\zeta_{c}^{d}, n+1\right)} .
$$

Combining this theorem with Lemma 4.1 immediately gives us the following:

Corollary 4.4. Let $\mathcal{F} \in H_{2-k}$ with $\xi_{2-k}(\mathcal{F}), D^{k-1}(\mathcal{F}) \in S_{k}^{!}$. Then we have for $0<n<k-2$

$$
L_{\xi_{2-k}(\mathcal{F})}(\chi, n+1)=(-1)^{n} \frac{(4 \pi)^{k-1}}{(k-2) !} \overline{L_{D^{k-1}(\mathcal{F})}\left(\chi^{-}, n+1\right)},
$$

where $\chi^{-}(m):=\chi(-m)$.

Proof of Theorem 4.3. Define

$$
\begin{aligned}
\mathcal{G}(z):= & -(2 i)^{1-k} R \cdot \int_{-\bar{z}}^{i \infty} \xi_{2-k}(\mathcal{F})^{c}(w)(z+w)^{k-2} d w \\
& +c_{k}^{-1} R \cdot \int_{z}^{i \infty} D^{k-1}(\mathcal{F})(w)(z-w)^{k-2} d w,
\end{aligned}
$$

where $g^{c}(z):=\sum_{m \geq m_{0}} \overline{a_{g}(m)} q^{m}$ for $g(z)=\sum_{m \geq m_{0}} a_{g}(m) q^{m} \in S_{k}^{!}$. We show that $\mathcal{G}$ and $\mathcal{F}$ are identical up to a constant term. A direct calculation gives us that

$$
\xi_{2-k}(\mathcal{G})=\xi_{2-k}(\mathcal{F}) .
$$

Moreover, using Lemma 2.3, we obtain that

$$
D^{k-1}(\mathcal{G})=D^{k-1}\left(\mathcal{E}_{D^{k-1}(\mathcal{F})}\right)=D^{k-1}(\mathcal{F}) .
$$

It follows that

$$
\mathcal{G}=\mathcal{F}+p
$$


where $p$ is a polynomial of degree at most $k-2$. Because $\mathcal{G}$ and $\mathcal{F}$ are both translation invariant, we have that $p=a_{0}$ is constant. Using the modularity of $\mathcal{F}$ with $\gamma=\left(\begin{array}{ll}a & b \\ c & d\end{array}\right) \in \mathrm{SL}_{2}(\mathbb{Z})$ we have that

$$
\begin{array}{r}
0=\left.\mathcal{F}\right|_{2-k}(1-\gamma)\left(z-\frac{d}{c}\right)=\left.\mathcal{F}_{1}\right|_{2-k}(1-\gamma)\left(z-\frac{d}{c}\right) \\
+\left.\mathcal{F}_{2}\right|_{2-k}(1-\gamma)\left(z-\frac{d}{c}\right) \\
-\left.a_{0}\right|_{2-k}(1-\gamma)\left(z-\frac{d}{c}\right)
\end{array}
$$

where

$$
\begin{aligned}
& \mathcal{F}_{1}(z):=-(2 i)^{1-k} R . \int_{-\bar{z}}^{i \infty} \xi_{2-k}(\mathcal{F})^{c}(w)(z+w)^{k-2} d w \\
& \mathcal{F}_{2}(z):=c_{k}^{-1} R . \int_{z}^{i \infty} D^{k-1}(\mathcal{F})(w)(z-w)^{k-2} d w .
\end{aligned}
$$

Clearly we have

$$
\left.a_{0}\right|_{2-k}(1-\gamma)\left(z-\frac{d}{c}\right)=a_{0}\left(1-(c z)^{k-2}\right) .
$$

Moreover, a direct calculation shows that

$$
\begin{aligned}
& \left.\mathcal{F}_{1}(z)\right|_{2-k} \gamma=-(2 i)^{1-k} R . \int_{-\bar{z}}^{\frac{d}{c}} \xi_{2-k}(\mathcal{F})^{c}(w)(z+w)^{k-2} d w \\
& \left.\mathcal{F}_{2}(z)\right|_{2-k} \gamma=c_{k}^{-1} R . \int_{z}^{-\frac{d}{c}} D^{k-1}(\mathcal{F})(w)(z-w)^{k-2} d w .
\end{aligned}
$$

Thus

$$
\begin{aligned}
& (2 i)^{1-k} R \cdot \int_{\frac{d}{c}}^{i \infty} \xi_{2-k}(\mathcal{F})^{c}(w)\left(z-\frac{d}{c}+w\right)^{k-2} d w \\
& \equiv c_{k}^{-1} R \cdot \int_{-\frac{d}{c}}^{i \infty} D^{k-1}(\mathcal{F})(w)\left(z-\frac{d}{c}-w\right)^{k-2} d w \quad\left(\bmod 1-(c z)^{k-2}\right) .
\end{aligned}
$$

Inserting the binomial expansion and using Theorem 4.2 then gives

$$
\begin{aligned}
& c_{k}^{-1} \sum_{n=0}^{k-2} i^{1-n}\left(\begin{array}{c}
k-2 \\
n
\end{array}\right) L_{D^{k-1}(\mathcal{F})}^{*}\left(\zeta_{c}^{-d}, n+1\right) z^{k-2-n} \\
& \equiv(2 i)^{1-k} \sum_{n=0}^{k-2} i^{1-n}\left(\begin{array}{c}
k-2 \\
n
\end{array}\right) L_{\xi_{2-k}(\mathcal{F})^{c}}^{*}\left(\zeta_{c}^{d}, n+1\right)(-z)^{k-2-n} \quad\left(\bmod 1-(c z)^{k-2}\right) .
\end{aligned}
$$

The claim now follows by comparing coefficients and using the fact that for integers $0 \leq n \leq k-2$ we have

$$
L_{f^{c}}^{*}\left(\zeta_{c}^{d}, n+1\right)=\overline{L_{f}^{*}\left(\zeta_{c}^{-d}, n+1\right)} .
$$

Proof of Theorem 1.2. Let $\mathcal{F} \in H_{2-k}$ with $f=\xi_{2-k}(\mathcal{F}) \in S_{k}^{!}$. By definition,

$$
\mathbb{P}\left(\mathcal{F}^{+}, \gamma_{c, d} ; z\right)=-\frac{(4 \pi)^{k-1}}{(k-2) !}\left(\mathcal{F}^{-}-\left.\mathcal{F}^{-}\right|_{2-k} \gamma_{c, d}\right)(z) .
$$


As in the proof of Theorem 4.3, we obtain

$$
\mathbb{P}\left(\mathcal{F}^{+}, \gamma_{c, d} ; z-\frac{d}{c}\right)=-\frac{(2 \pi i)^{k-1}}{(k-2) !} \sum_{n=0}^{k-2}\left(\begin{array}{c}
k-2 \\
n
\end{array}\right) i^{n+1} L_{f^{c}}^{*}\left(\zeta_{c}^{d}, n+1\right) z^{k-2-n} .
$$

Next we use (4.2) to find that

$$
\begin{aligned}
\overline{\mathbb{P}\left(\mathcal{F}^{+}, \gamma_{c, d} ; \bar{z}-\frac{d}{c}\right)} & =\frac{(2 \pi i)^{k-1}}{(k-2) !} \sum_{n=0}^{k-2}\left(\begin{array}{c}
k-2 \\
n
\end{array}\right) i^{-n-1} L_{f}^{*}\left(\zeta_{c}^{-d}, n+1\right) z^{k-2-n} \\
& =\sum_{n=0}^{k-2} \frac{L_{f}\left(\zeta_{c}^{-d}, n+1\right)}{(k-2-n) !}(2 \pi i z)^{k-2-n} .
\end{aligned}
$$

Finally, we apply Lemma 4.1 to obtain the result.

\section{Proof of Theorem 1.1}

We begin by recalling some basic facts regarding the incomplete gamma function (see Section 8 of [17] for more details). For $s \in \mathbb{C}$ with $\operatorname{Re}(s)>0$, we define the incomplete gamma function by

$$
\Gamma(s, x):=\int_{x}^{\infty} e^{-t} t^{s} \frac{d t}{t} .
$$

We note that the usual gamma function is given for $\operatorname{Re}(s)>0$ by $\Gamma(s):=\Gamma(s, 0)$. Integration by parts gives the following recurrence relation:

$$
\Gamma(s+1, x)=e^{-x} x^{s}+s \Gamma(s, x) .
$$

There is an analytic continuation of $\Gamma(s, x)$ to an entire function with respect to $s$ for fixed $x \neq 0$ (see Section 8.2 of [17]). Note that (5.1) also holds for $x>0$ and $s \in \mathbb{C}$. Moreover, for $n \in \mathbb{N}$, we require the following representations:

$$
\frac{\Gamma(n, x)}{(n-1) !}=e^{-x} \sum_{m=0}^{n-1} \frac{x^{m}}{m !}
$$

and

$$
\Gamma(-n, x)=(-1)^{n} \frac{\Gamma(0, x)}{n !}+\frac{(-1)^{n+1} e^{-x}}{n ! x} \sum_{m=0}^{n-1} \frac{(-1)^{m} m !}{x^{m}} .
$$

A main step in the proof of Theorem 1.1 is the following.

Proposition 5.1. Let $\delta=0$ or 1 , and $f(z)=\sum_{m \geq m_{0}} a_{f}(m) q^{m} \in S_{k}^{!}$. Then

$$
\lim _{\substack{n \rightarrow \infty \\ n=\delta(\bmod 2)}} \frac{\left(2 \pi m_{1}\right)^{n}}{(n-1) !} L_{f}^{*}(n)=a_{f}\left(m_{1}\right)+(-1)^{\delta} a_{f}\left(-m_{1}\right),
$$

where $m_{1} \in \mathbb{N}$ is minimal with

$$
a_{f}\left(m_{1}\right)+(-1)^{\delta} a_{f}\left(-m_{1}\right) \neq 0 .
$$

Proof. We consider the individual pieces of the $L$-series (1.5) for $f$ and choose $t_{0}=1$. First we show that

$$
\lim _{n \rightarrow \infty} \sum_{m \geq m_{0}} a_{f}(m) \frac{\Gamma(k-n, 2 \pi m)}{(2 \pi m)^{k-n}}=\lim _{n \rightarrow \infty} \sum_{m \geq m_{0}} a_{f}(m) \Gamma(-n, 2 \pi m)(2 \pi m)^{n}
$$


exists. This then clearly gives us that the corresponding contribution to the limit is 0 since for all $x$

$$
\lim _{n \rightarrow \infty} \frac{x^{n}}{n !}=0 .
$$

Firstly we split off all negative indexed terms. For this, we show that for $|x|>1$ the function $\Gamma(-n, x) x^{n}$ is bounded. Using (5.3), we estimate

$$
\left|\Gamma(-n, x) x^{n}\right| \ll \frac{|x|^{n}}{n !} \sum_{m=0}^{n-1} \frac{m !}{|x|^{m}}=\sum_{m=0}^{n-1}\left(\begin{array}{c}
n \\
m
\end{array}\right)^{-1} \frac{|x|^{n-m}}{(n-m) !} \leq \sum_{m=1}^{n} \frac{|x|^{m}}{m !}<e^{x} .
$$

Here all bounds depend on $x$.

We next show that

$$
\lim _{n \rightarrow \infty} \sum_{m \geq 1} a_{f}(m) \Gamma(-n, 2 \pi m)(2 \pi m)^{n}=0 .
$$

Using (1.6) gives us that the sum on $m$ for some $c>0$ may be bounded against

$$
\begin{aligned}
& \ll \sum_{m \geq 1}(2 \pi m)^{n+\frac{1}{2}\left(k-\frac{3}{2}\right)} e^{c \sqrt{2 \pi m}} \Gamma(-n, 2 \pi m) \ll \int_{2 \pi}^{\infty} \Gamma(-n, x) x^{n+\frac{1}{2}\left(k-\frac{3}{2}\right)} e^{c \sqrt{x}} d x \\
& =\int_{2 \pi}^{\infty} x^{\frac{1}{2}\left(k-\frac{3}{2}\right)} e^{c \sqrt{x}} \int_{1}^{\infty} e^{-t x} t^{-n-1} d t d x .
\end{aligned}
$$

Using integration by parts, the inner integral may be estimated against

$$
\frac{e^{-x}}{n}-\frac{x}{n} \int_{1}^{\infty} e^{-t x} t^{-n} d t \ll \frac{e^{-x}}{n}+\frac{x e^{-x}}{n} \int_{0}^{\infty} e^{-t x} d t \ll \frac{e^{-x}}{n} .
$$

Thus (5.6) can be bounded by

$$
\frac{1}{n} \int_{2 \pi}^{\infty} e^{-x+c \sqrt{x}} d x \ll \frac{1}{n} \stackrel{n \rightarrow \infty}{\rightarrow} 0,
$$

giving (5.5).

We next show that for $M>\frac{2 c^{2}}{\pi}$ we have that

$$
\sum_{m \geq M} \frac{a_{f}(m) \Gamma(n, 2 \pi m)}{(2 \pi m)^{n}} \ll \frac{(n-2) !}{(\pi M)^{n}} .
$$

Picking $M>2 m_{1}$ then gives us that the corresponding contribution to the limit vanishes. To see (5.7) we again use (1.6) to bound the left hand side against

$$
\begin{aligned}
& \ll(2 \pi)^{-n} \sum_{m \geq M} m^{\frac{1}{2}\left(k-\frac{3}{2}\right)-n} e^{c \sqrt{2 \pi m}} \int_{2 \pi m}^{\infty} e^{-t} t^{n-1} d t \\
& \ll(2 \pi)^{-n} \sum_{m \geq M} m^{\frac{1}{2}\left(k-\frac{3}{2}\right)-n} \int_{2 \pi m}^{\infty} e^{-\frac{t}{2}} t^{n-1} d t \\
& \ll(2 \pi)^{-n} \sum_{m \geq M} m^{\frac{1}{2}\left(k-\frac{3}{2}\right)-n} \int_{0}^{\infty} e^{-\frac{t}{2}} t^{n-1} d t \\
& \ll \pi^{-n}(n-1) ! \sum_{m \geq M} m^{\frac{1}{2}\left(k-\frac{3}{2}\right)-n} \ll \frac{(n-2) !}{(\pi M)^{n}},
\end{aligned}
$$

as claimed. 
For the remaining terms, we combine the $m$-th and the $-m$-th terms. For $n \equiv \delta$ $(\bmod 2)$ we have that

$$
\begin{aligned}
& \frac{\left(2 \pi m_{1}\right)^{n}}{(n-1) !}\left(a_{f}(m) \frac{\Gamma(n, 2 \pi m)}{(2 \pi m)^{n}}+a_{f}(-m) \frac{\Gamma(n,-2 \pi m)}{(-2 \pi m)^{n}}\right) \\
& =\left(\frac{m_{1}}{m}\right)^{n}\left(a_{f}(m) \frac{\Gamma(n, 2 \pi m)}{(n-1) !}+(-1)^{\delta} a_{f}(-m) \frac{\Gamma(n,-2 \pi m)}{(n-1) !}\right) .
\end{aligned}
$$

Using the fact (which easily follows from (5.2)) that for all real $x$,

$$
\lim _{n \rightarrow \infty} \frac{\Gamma(n, x)}{(n-1) !}=1,
$$

we then obtain for $m \geq m_{0}$

$$
\begin{aligned}
& \lim _{\substack{n \rightarrow \infty \\
n \equiv \delta(\bmod 2)}} \frac{\left(2 \pi m_{1}\right)^{n}}{(n-1) !}\left(a_{f}(m) \frac{\Gamma(n, 2 \pi m)}{(2 \pi m)^{n}}+a_{f}(-m) \frac{\Gamma(n,-2 \pi m)}{(-2 \pi m)^{n}}\right) \\
& =\left(a_{f}(m)+(-1)^{\delta} a_{f}(-m)\right) \lim _{\substack{n \rightarrow \infty \\
n \equiv \delta(\bmod 2)}}\left(\frac{m_{1}}{m}\right)^{n} \\
& = \begin{cases}a_{f}\left(m_{1}\right)+(-1)^{\delta} a_{f}\left(-m_{1}\right) & \text { if } m=m_{1}, \\
0 & \text { otherwise. }\end{cases}
\end{aligned}
$$

For $0<m<m_{1}$, we have

$$
(-1)^{\delta} a_{f}(-m)=-a_{f}(m)
$$

by hypothesis. Using this reduces (5.8) to

$$
a_{f}(m)\left(\frac{m_{1}}{m}\right)^{n} \frac{\Gamma(n, 2 \pi m)-\Gamma(n,-2 \pi m)}{(n-1) !} .
$$

To finish the proof, it suffices to show that (5.9) vanishes as $n \rightarrow \infty$. To see this, we bound for $x>1$

$$
\Gamma(n, x)-\Gamma(n,-x)=\int_{x}^{-x} e^{-t} t^{n-1} d t \ll x^{n} .
$$

Thus (5.9) may be estimated against

$$
\ll\left(\frac{m_{1}}{m}\right)^{n} \frac{(2 \pi m)^{n}}{(n-1) !} \stackrel{n \rightarrow \infty}{\rightarrow} 0,
$$

again using (5.4).

Corollary 5.2. If $f \in S_{k}^{!}, f \not \equiv 0$, then

$$
\lim _{n \rightarrow \infty}\left|L_{f}^{*}(n)\right|=\infty \text {. }
$$

Corollary 5.3. For $f \in S_{k}^{!}, f \not \equiv 0$, at most finitely many values $L_{f}^{*}(n)$ vanish for $n \in \mathbb{N}$.

Corollary 5.4. Let $f, g \in S_{k}^{!}$with $g \not \equiv 0$ and q-expansions given by $f(z)=$ $\sum_{m \geq m_{0}} a_{f}(m) q^{m}$ and $g(z)=\sum_{m \geq m_{0}} a_{g}(m) q^{m}$. For $\delta=0$ or 1 we have that

$$
\lim _{n \equiv \delta \rightarrow \infty} \frac{L_{f}^{*}(n)}{(\bmod 2)}=\frac{a_{f}\left(m_{1}\right)+(-1)^{\delta} a_{f}\left(-m_{1}\right)}{L_{g}^{*}(n)},
$$

where $m_{1}$ is the smallest positive integer such that at least one of the numerator or denominator on the right hand side is nonzero. 
Remark. The right hand side is taken to be infinity whenever the denominator is zero.

Proof of Theorem 1.1. Since $\mathcal{F} \in H_{2-k}^{*}$ is good for $g$, the $q$-expansion of $\xi_{2-k}(\mathcal{F})$ is

$$
\xi_{2-k}(\mathcal{F})(z)=\langle g, g\rangle^{-1} \sum_{m=1}^{\infty} a_{g}(n) q^{m},
$$

with $a_{g}(1)=1$. Moreover, by Corollary 5.4 , it follows that

$\lim _{\substack{n \equiv \delta \\ n \rightarrow \infty \bmod 2)}} \frac{L_{D^{k-1}(\mathcal{F})}(n)}{L_{\xi_{2-k}(\mathcal{F})}(n)}=\lim _{\substack{n \equiv \delta \rightarrow \infty \\ n \rightarrow(\bmod 2)}} \frac{L_{D^{k-1}(\mathcal{F})}^{*}(n)}{L_{\xi_{2-k}(\mathcal{F})}^{*}(n)}=\left(c_{\mathcal{F}}^{+}(1)+(-1)^{\delta} c_{\mathcal{F}}^{+}(-1)\right)\langle g, g\rangle$.

The result may now be concluded since we have $\langle g, g\rangle=\omega_{g}^{+} \omega_{g}^{-}$.

\section{REFERENCES}

[1] Spencer Bloch and Kazuya Kato, L-functions and Tamagawa numbers of motives, The Grothendieck Festschrift, Vol. I, Progr. Math., vol. 86, Birkhäuser Boston, Boston, MA, 1990, pp. 333-400. MR:1086888 (92g:11063)

[2] Kathrin Bringmann, Pavel Guerzhoy, Zachary Kent, and Ken Ono, Eichler-Shimura theory for mock modular forms, Math. Ann. 355 (2013), no. 3, 1085-1121, DOI 10.1007/s00208012-0816-y. MR3020155

[3] Jan Hendrik Bruinier and Jens Funke, On two geometric theta lifts, Duke Math. J. 125 (2004), no. 1, 45-90, DOI 10.1215/S0012-7094-04-12513-8. MR2097357(2005m:11089)

[4] Jan H. Bruinier, Ken Ono, and Robert C. Rhoades, Differential operators for harmonic weak Maass forms and the vanishing of Hecke eigenvalues, Math. Ann. 342 (2008), no. 3, 673-693, DOI 10.1007/s00208-008-0252-1. MR2430995(2009f:11046)

[5] Daniel Bump, Solomon Friedberg, and Jeffrey Hoffstein, Nonvanishing theorems for Lfunctions of modular forms and their derivatives, Invent. Math. 102 (1990), no. 3, 543-618, DOI 10.1007/BF01233440. MR1074487 (92a:11058)

[6] P. Deligne, Valeurs de fonctions $L$ et périodes d'intégrales (French), with an appendix by N. Koblitz and A. Ogus, Automorphic forms, representations and $L$-functions (Proc. Sympos. Pure Math., Oregon State Univ., Corvallis, Ore., 1977), Part 2, Proc. Sympos. Pure Math., XXXIII, Amer. Math. Soc., Providence, R.I., 1979, pp. 313-346. MR.546622 (81d:12009)

[7] Benedict H. Gross and Don B. Zagier, Heegner points and derivatives of L-series, Invent. Math. 84 (1986), no. 2, 225-320, DOI 10.1007/BF01388809. MR833192 (87j:11057)

[8] P. Guerzhoy, Hecke operators for weakly holomorphic modular forms and supersingular congruences, Proc. Amer. Math. Soc. 136 (2008), no. 9, 3051-3059, DOI 10.1090/S0002-993908-09277-0. MR2407067 (2009e:11089)

[9] Pavel Guerzhoy, Zachary A. Kent, and Ken Ono, p-adic coupling of mock modular forms and shadows, Proc. Natl. Acad. Sci. USA 107 (2010), no. 14, 6169-6174, DOI 10.1073/pnas.1001355107. MR2630103 (2011h:11048)

[10] Henryk Iwaniec, On the order of vanishing of modular L-functions at the critical point, Sém. Théor. Nombres Bordeaux (2) 2 (1990), no. 2, 365-376. MR1081731 (92h:11040)

[11] N. I. Koblic, Non-integrality of the periods of cusp forms outside the critical strip (Russian), Funkcional. Anal. i Priložen. 9 (1975), no. 3, 52-55. MR0404144 (53 \#7948)

[12] W. Kohnen and D. Zagier, Modular forms with rational periods, Modular forms (Durham, 1983), Ellis Horwood Ser. Math. Appl.: Statist. Oper. Res., Horwood, Chichester, 1984, pp. 197-249. MR803368 (87h:11043)

[13] W. Kohnen and D. Zagier, Values of L-series of modular forms at the center of the critical strip, Invent. Math. 64 (1981), no. 2, 175-198, DOI 10.1007/BF01389166. MR629468 (83b:10029)

[14] V. A. Kolyvagin, Finiteness of $E(\mathbf{Q})$ and $S H(E, \mathbf{Q})$ for a subclass of Weil curves (Russian), Izv. Akad. Nauk SSSR Ser. Mat. 52 (1988), no. 3, 522-540, 670-671; English transl., Math. USSR-Izv. 32 (1989), no. 3, 523-541. MR954295 (89m:11056)

[15] Maxim Kontsevich and Don Zagier, Periods, Mathematics unlimited-2001 and beyond, Springer, Berlin, 2001, pp. 771-808. MR.1852188 (2002i:11002) 
[16] Ju. I. Manin, Periods of cusp forms, and p-adic Hecke series (Russian), Mat. Sb. (N.S.) 92(134) (1973), 378-401, 503. MR0345909 (49 \#10638)

[17] Digital Library of Mathematical Functions, National Institute of Standards and Technology, from http://dlmf.nist.gov/, 2011.

[18] Ken Ono, Unearthing the visions of a master: harmonic Maass forms and number theory, Current developments in mathematics, 2008, Int. Press, Somerville, MA, 2009, pp. 347-454. MR:2555930(2010m:11060)

[19] Ken Ono and Christopher Skinner, Fourier coefficients of half-integral weight modular forms modulo l, Ann. of Math. (2) 147 (1998), no. 2, 453-470, DOI 10.2307/121015. MR 1626761 (99f:11059a)

[20] Ken Ono and Christopher Skinner, Non-vanishing of quadratic twists of modular L-functions, Invent. Math. 134 (1998), no. 3, 651-660, DOI 10.1007/s002220050275. MR 1660945 (2000a:11077)

[21] Norbert Schappacher and Anthony J. Scholl, Beilinson's theorem on modular curves, Berlinson's conjectures on special values of $L$-functions, Perspect. Math., vol. 4, Academic Press, Boston, MA, 1988, pp. 273-304. MR944997 (89h:11029)

[22] D. Zagier, Ramanujan's mock theta functions and their applications (after Zwegers and OnoBringmann), Sém. Bourbaki, 60éme année, Vol. 2007/2008, Astérisque No. 326 (2009), Exp. No. 986, 143-164. MR2605321

[23] S. P. Zwegers, Mock $\theta$-functions and real analytic modular forms, $q$-series with applications to combinatorics, number theory, and physics (Urbana, IL, 2000), Contemp. Math., vol. 291, Amer. Math. Soc., Providence, RI, 2001, pp. 269-277, DOI 10.1090/conm/291/04907. MR.1874536 (2003f:11061)

[24] S. Zwegers, Mock theta functions, Ph.D. Thesis, University of Utrecht, 2002.

Mathematical Institute, University of Cologne, Weyertal 86-90, 50931 Cologne, GERMANY

E-mail address: kbringma@math.uni-koeln.de

Max-Planck-Institut für Mathematik, Vivatsgasse 7, 53111, Bonn, Germany

E-mail address: fricke.karl-heinz@freenet.de

Department of Mathematics and Computer Science, Emory University, Atlanta, Georgia 30322

E-mail address: kent@mathcs.emory.edu 